\title{
Giant cell arteritis: A rare cause of posterior vasculitis
}

\author{
Marilita M Moschos' \\ Yan Guex-Crosier ${ }^{2}$ \\ 'Department of Ophthalmology, \\ University of Athens, Greece; \\ ${ }^{2}$ Jules Gonin Eye Hospital, University \\ of Lausanne, Switzerland
}

Purpose: To report three cases of posterior vasculitis associated with subacute giant cell arteritis (GCA).

Methods: Three patients with decreased vision underwent complete ophthalmologic examination and fluorescein angiography.

Results: All patients presented posterior vasculitis. Patient 1 had an erythrocyte sedimentation rate (ESR) of $38 \mathrm{~mm} / \mathrm{hr}$ and a C-reactive protein (CRP) of $28 \mathrm{mg} / \mathrm{L}$. Patient 2 and 3 had an ESR of 104 and $95 \mathrm{~mm} / \mathrm{hr}$ and a CRP of 42 and $195 \mathrm{mg} / \mathrm{L}$ accordingly. Diagnosis was established by temporal artery biopsy. Resolution was observed after systemic prednisolone therapy.

Conclusion: GCA should be suspected when posterior vasculitis and relatively high ESR and CRP are present.

Keywords: giant cell arteritis, posterior vasculitis Horton's disease, temporal arteritis, uveitis

\section{Introduction}

Giant cell arteritis (GCA), known as temporal arteritis or Horton's disease is a systemic vasculitis of unknown etiology and pathogenesis, which typically occurs in patients older than 60 years. ${ }^{1}$ The typical ophthalmic symptom is sudden vision loss in one or both eyes. In the majority of cases the diagnosis of GCA is based on a positive temporal artery biopsy result. In approximately $10 \%$ of patients, in whom the biopsy is falsely negative the diagnosis can be based on the clinical features.

Posterior vasculitis associated with low-grade uveitis is uncommon as a presenting feature of GCA.

We present three cases of GCA who had developed posterior vasculitis. In all cases GCA could be proven by temporal artery biopsy.

\section{Case reports}

\section{Patient I}

A 70-year-old man was referred to the Jules Gonin Eye Hospital for cataract extraction. Four months before, he complained of a rapid decrease of visual acuity (VA) of OS, associated with violent headaches. A partial occlusion of the central artery of the retina of the OD was then diagnosed by his ophthalmologist.

At presentation best-corrected VA measured with a standard Snellen chart was found equal to $0.2 \mathrm{OD}$ and light perception (LP) OS. There was a left afferent pupillary defect. Slit lamp examination revealed the presence of a bilateral cataract. The fundus examination revealed the presence of inflammatory cells in the vitreous, right disc edema, multiple raised creamy subretinal peripapillary lesions and narrow arterial vessels of the posterior pole. The fluorescein angiography revealed signs of posterior vasculitis with fluffy, white perivascular infiltrates in the eye (Figure 1). Also, visual fields were affected in the OD with an altitudinal defect. 


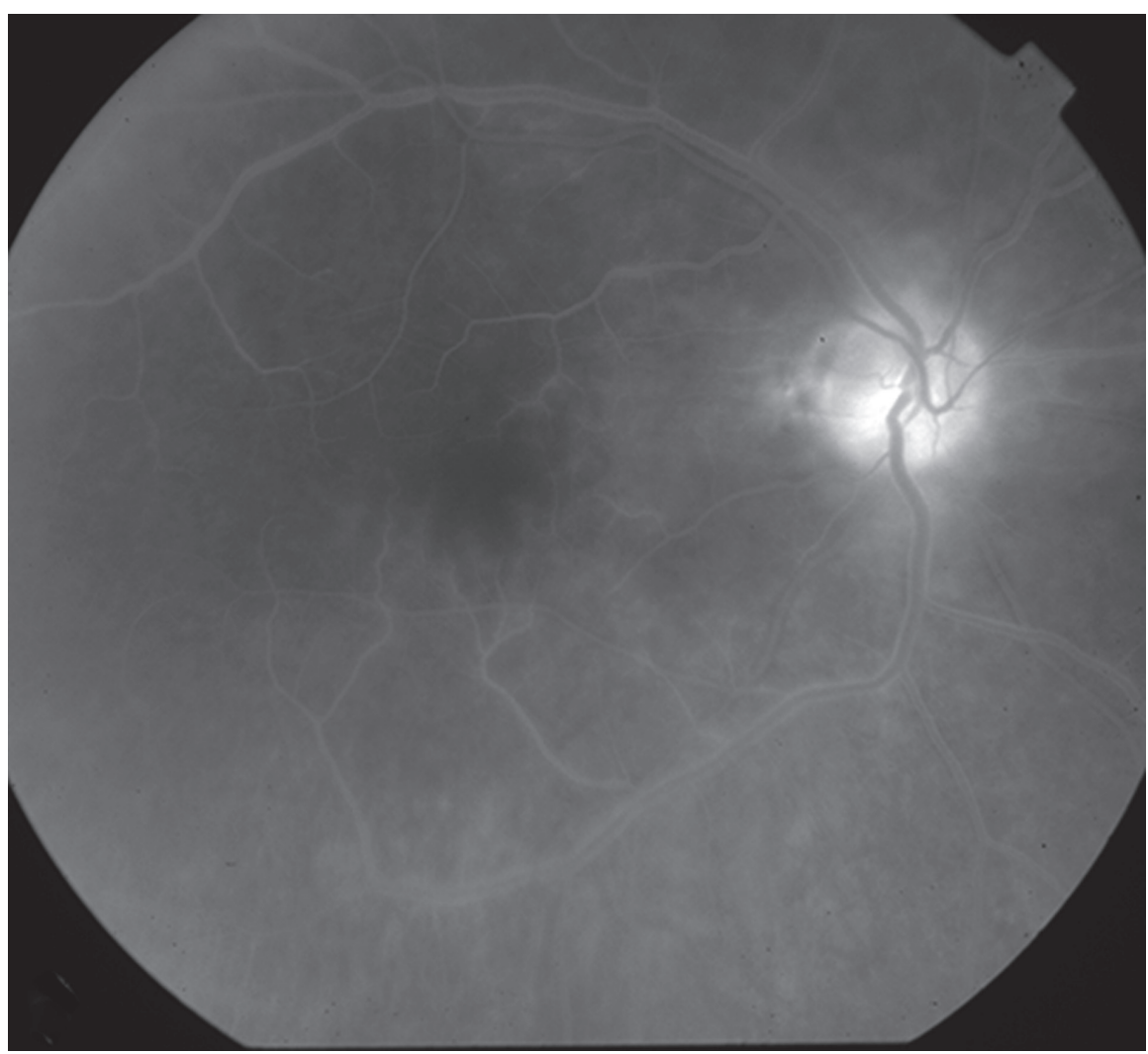

Figure I Fluorescein angiography OD showing leakage from the subretinal peripapillary lesions and the optic disc with vasculitis of the posterior pole.

The general physical examination was essentially normal. Investigations revealed an erythrocyte sedimentation rate (ESR) of $38 \mathrm{~mm} / \mathrm{hr}$ in the first hour and $78 \mathrm{~mm} / \mathrm{hr}$ in the second hour along with a C-reactive protein (CRP) of $28 \mathrm{mg} / \mathrm{ml}$ (normal value $<6 \mathrm{mg} / \mathrm{ml}$ ). A temporal artery biopsy was performed which showed evidence of GCA.

With the diagnosis of GCA, systemic steroid therapy was initiated. Eight months later cataract surgery was performed, while the patient was still treated with prednisolone $20 \mathrm{mg} / \mathrm{d}$ orally administered. Final best-corrected VA improved to 0.4 in the OD.

\section{Patient 2}

An 83-year-old woman was referred to our department with the diagnosis of bilateral cataract and anterior synechiae of the OS. Nine months earlier, the patient described increased fatigability, bitemporal severe headaches and anorexia with a 6-kg weight loss. Three months after the onset of symptoms, VA started to decrease in both eyes.

At presentation, best-corrected VA measured with a standard Snellen chart was 0.05 OD and 0.7 OS. No afferent pupillary defect was present. Ocular motility was normal. Goldmann visual fields showed a superior temporal loss in the OD. The left visual field was normal. Fundus examination showed a swollen right optic disc. Fluorescein angiography revealed segmental disc hyperfluorescence and signs of arterial vasculitis at the posterior pole with arteriolar attenuation, sheathing and cotton wool spots suggestive of arteriolar involvement (Figure 2).

Investigations revealed the presence of hypochromic microcytic anaemia, diverticulitis along with an ESR of $104 \mathrm{~mm} / \mathrm{hr}$ in the first hour and a CRP of $42 \mathrm{mg} / \mathrm{ml}$. A temporal artery biopsy specimen confirmed GCA.

Under this diagnosis, methylprednisolone $1 \mathrm{mg} / \mathrm{kg} /$ day was administered intravenously for three days, reducing the ESR from 104 to the range of 84. At discharge, the patient's regimen consisted of prednisone $50 \mathrm{mg}$ orally per day. Two weeks later, the ESR was found equal to $18 \mathrm{~mm} / \mathrm{hr}$ in the first hour. Final best-corrected VA was 0.2 OD and 0.7 OS.

\section{Patient 3}

An 83-year-old man complained of rapid decrease of vision of the OS, diplopia and a pain in the left hemiface starting near the ear region with irradiation to the left temple. At that time, diagnosis of trigeminal neuralgia was suspected and investigations were focused on the temporo-mendibular 


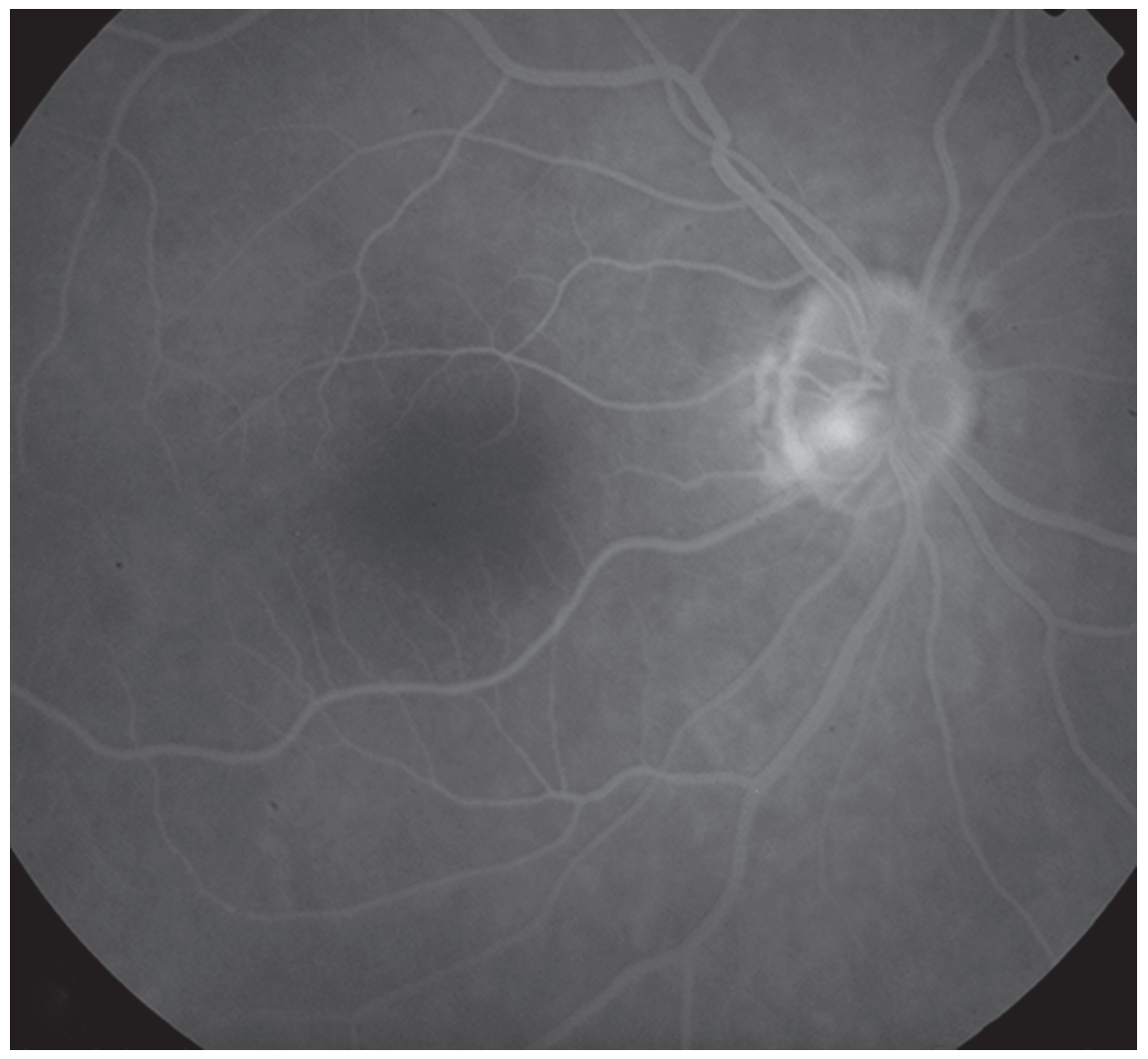

Figure 2 Fluorescein angiography OD showing partial disc edema and fine vasculitis of the posterior pole.

articulation. Two weeks later, a visual impairment of the OS occurred and the patient was referred to our department for further investigation.

At presentation, best-corrected VA was limited to count fingers for both eyes.

Fundus examination revealed the presence of pale optic nerve heads, narrowed arterial vessels and severe ischemia in both eyes. A zone of hyperfluorescence was present in the late phase fluorescein angiogram in the OD, secondary to posterior vasculitis (Figure 3).

Laboratory results revealed an ESR of $95 \mathrm{~mm} / \mathrm{hr}$ in the first hour and a CRP of $195 \mathrm{mg} / \mathrm{ml}$. A temporal artery biopsy specimen confirmed GCA.

Under this diagnosis, intravenous treatment with $500 \mathrm{mg}$ of methylprednisolone for three consecutive days, increased to $1 \mathrm{~g}$ for three more days was introduced. To avoid the onset of neovascularization a photocoagulation of the ischemic zones was performed. Systemic steroids were gradually tapered over the next few months and azathioprime was initiated, in order to decrease more rapidly the dose of steroids due to the appearance of diabetes mellitus.

At the end of the follow-up in our department, final best-corrected VA improved to 0.1 OD and 0.05 OS.
The patient was still treated with prednisone $15 \mathrm{mg} /$ day associated with azathioprime $150 \mathrm{mg} /$ day.

\section{Discussion}

GCA is a systemic vasculitis of unknown cause, which involves predominantly medium-sized extracranial arteries of the carotid circulation and sometimes the aorta and its primary branches. It can be distinguished from other vasculities by the age of the patient, the new onset of localized headache, a temporal artery tenderness or reduced pulsation, an elevated ESR, and characteristic findings at temporal artery biopsy. The rate of ophthalmic involvement is reported to be as high as $50 \%$ in some older series. ${ }^{2} \mathrm{~A}$ rapid partial or complete visual loss is the most common and the most serious ophthalmic complication of GCA. Rates of vision loss as high as $35 \%$ to $50 \%$ have been reported. ${ }^{3,4}$ Generally, vision loss from GCA is permanent. An elevated ESR and elevated levels of other acute-phase reactants such as fibrinogen, CRP, and haptoglobin are present in the vast majority of patients (patient 2 and 3).

This well known acute evolution of GCA, which involves typical cranial and ophthalmic signs and symptoms, should not occult the possibility of subacute or chronic forms of 


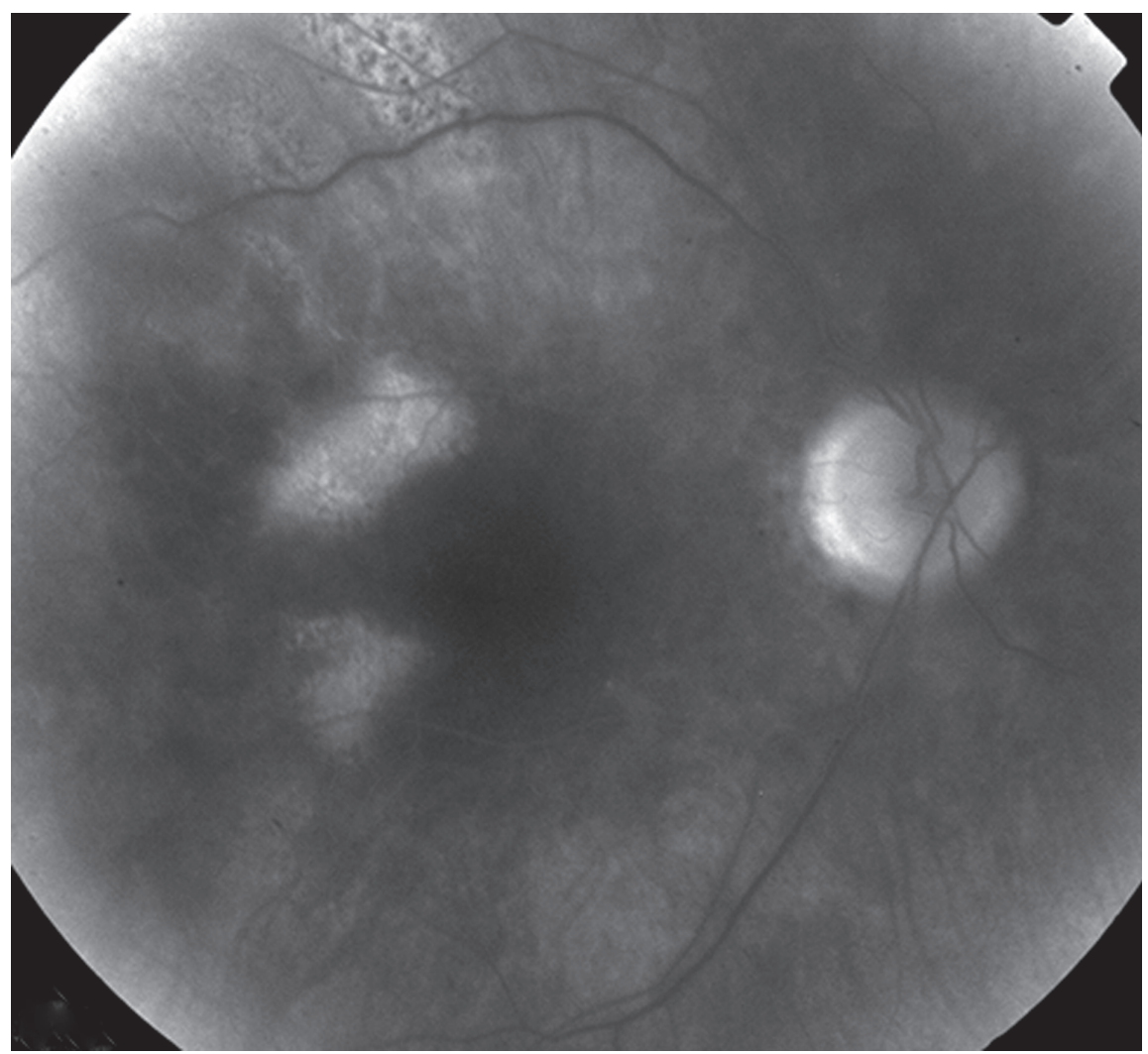

Figure 3 Fluorescein angiography OD reveals the hyperfluorescence of the optic disc due to vasculitis.

Horton's disease. A normal blood count and ESR do not exclude the diagnosis (patient 1). ${ }^{5,6}$

In our case series, all three patients had a subacute evolution of the disease of one month duration or more before the diagnosis and the onset of steroid therapy. Two (patient 2 and 3) were investigated in internal medicine, but in the presence of an atypical presentation, the diagnosis of GCA could not be promptly established. Although patient 2 had a history of weight loss and violent headaches at presentation, the diagnosis of GCA was not considered because many patients do not seek medical attention for headache or constitutional symptoms, and thus GCA remains undiagnosed until catastrophic vision loss occurs.

In patient 3 , the presence of sigmoidal diverticulitis was suspected to be at the origin, because sometimes clinicians elicit a more specific complaint such as tenderness or jaw claudication in order to pose the diagnosis of GCA.

In conclusion, because GCA is a potentially blinding disease and its early diagnose is the key to preventing blindness, it is important to recognize its early ocular manifestations. ${ }^{7}$ Posterior vasculitis as a presenting feature is uncommon and to the best of our knowledge there is one case report where the presenting feature was subacute panuveitis as a presenting feature of GCA. ${ }^{8}$ The fluorescein angiogram showed retinal vasculitis and there was no wedge shaped alteration suggestive of choroidal ischemia. ${ }^{9}$

In our case series, all three patients presented posterior vasculitis. Anterior synechiae (patient 3 ) can also be observed in cases of Horton's disease. A mild type of Horton's disease should also be kept in mind in cases presenting a chronic evolution with loss of weight, headache, or visual symptoms. In all of our cases of Horton's disease, the diagnosis could undoubtedly be established with a positive biopsy of the temporal artery.

\section{Conclusion}

We describe a case series of three patients in which GCA offers a possible explanation for the clinical picture of posterior vasculitis. Therefore, Horton's disease must absolutely be ruled out in cases of chronic ocular inflammation in order to avoid an irreversible severe vision impairment or vision loss.

\section{Disclosure}

The authors declare no conflicts of interest in this work. 


\section{References}

1. Hunder GC, Bloch DA, Michel BA, et al. The American College of Rheumatology 1990 criteria for the classification of giant cell arteritis. Arthritis Rheum. 1990;33:1122-1128.

2. Whitfield AGW, Bateman M, Trevor-Cooke W. Temporal arteritis. Br J Ophthalmol. 1963;47:555-566.

3. Birkhead NC, Wagener HP, Shick RM. Treatment of temporal arteritis with adrenal corticosteroids. JAMA. 1975;163:821-827.

4. Graham E, Holland A, Avery A, et al. Prognosis in giant-cell arteritis. Br Med J. 1981;282:269-271.

5. Anderson R, Malmvall B, Bengsson B. Acute phase reactants in the initial phase of giant cell arteritis. Acta Med Scand. 1986;220:365-367.
6. Kyle V, Cawston TE, Hazleman BL. Erythrocyte sedimentation rate and $\mathrm{C}$ reactive protein in the assessment of polymyalgia rheumatica/giant cell arteritis on presentation and during follow-up. Ann Rheum Dis. 1989;48:667-671.

7. Hayreh SS, Podhajsky PA, Zimmerman B. Ocular manifestations of giant cell arteritis. J Ophthalmol. 1998;125:509-520.

8. Rajesh CV. Panuveitis as a presenting feature of giant cell arteritis. Br J Ophthalmol. 2000;84:340.

9. Amalric P. Acute choroidal ischaemia. Trans Ophthalmol Soc UK. 1971;91:305-322 
